\title{
Loss of Heterozygosity in Fibrocystic Change of the Breast
}

\section{Genetic Relationship Between Benign Proliferative Lesions and Associated Carcinomas}

\author{
Constance Washington, ${ }^{\dagger}$ Fabienne Dalbègue, ${ }^{*}$ \\ Fleurette Abreo, ${ }^{\dagger}$ Jeffery K. Taubenberger, ${ }^{*}$ and \\ Jack H. Lichy* \\ From the Molecular Pathology Division," Department of Cellular \\ Pathology, Armed Forces Institute of Pathology, Washington, \\ D.C.; and the Department of Pathology, ${ }^{\dagger}$ Louisiana State \\ University Medical Center, Shreveport, Louisiana
}

Loss of heterozygosity (LOH), a genetic change frequently detected in cancer, can also occur in benign epithelial foci in the breast. To characterize LOH in benign breast tissue, 32 cases containing the various components of fibrocystic change in the absence of malignancy were studied. Microdissected foci of ductal hyperplasia, apocrine metaplasia, sclerosing adenosis, and morphologically normal terminal duct lobular units (TDLUs) were analyzed for LOH at 14 polymorphic loci representing seven chromosomal arms. LOH was detected in $22 \%$ of normal TDLUs $(6 / 27), 17 \%$ of adenosis $(4 / 23), 19 \%$ of hyperplasia $(4 / 21)$, and $53 \%$ of apocrine metaplasia (10/19) specimens. Because of the high percentage of $\mathrm{LOH}$ in apocrine metaplasia in nonneoplastic specimens, the genetic relationship between apocrine metaplasia and cancer was studied in a panel of breast cancer cases. Of 14 examples of apocrine metaplasia adjacent to a carcinoma, seven were found to have $\mathrm{LOH}$ with at least one marker. In all seven cases, the tumor and apocrine metaplasia shared LOH at one or more markers. The results demonstrate that LOH occurs frequently in the components of fibrocystic change as well as in normal TDLUs and suggest that foci of apocrine metaplasia can share a genetically altered precursor cell with an associated carcinoma. (Am J Pathol 2000, 157:323-329)

Recent observations of loss of heterozygosity $(\mathrm{LOH})$ in benign breast epithelium raise questions regarding the occurrence of this phenomenon in the physiological development of the breast and the relationship of $\mathrm{LOH}$ in normal breast epithelium to that seen in cancer. $\mathrm{LOH}$ has now been observed in benign terminal duct lobular units (TDLUs) adjacent to cancer ${ }^{1}$ and in proliferative lesions believed to be premalignant, such as atypical hyperplasia and carcinoma in situ. ${ }^{2-6}$ In addition, $\mathrm{LOH}$ has been reported to occur in isolated epithelial foci not specifically associated with malignancy, including the components of fibrocystic change. An examination of microdissected TDLUs from reduction mammoplasty and benign excision biopsy specimens has demonstrated that even in the absence of adjacent pathological proliferative lesions $\mathrm{LOH}$ can be detected in benign lobules, albeit with a much lower frequency than that observed in malignant tumors of the breast.

The relative risk for the development of cancer associated with specific benign pathological entities has been characterized in epidemiological studies. ${ }^{8,9}$ The presence of mild sclerosing adenosis, apocrine metaplasia, mild hyperplasia, fibrosis, cysts, or a solitary papilloma carries no increased risk of cancer. Florid or atypical hyperplasia leads to a moderate increase (1.5-4 times) in the relative risk, and lobular or ductal carcinoma in situ increases the risk for cancer by 8-10 times. For each of these pathological findings, including those associated with increased cancer risk, it is generally not known whether the abnormal proliferation is an actual precursor

Supported by grant DAMD 17-94-J-4330 from the U.S. Army Medical Research and Materiel Command and by the intramural funds of the Armed Forces Institute of Pathology. C. W. and F. A. were supported by the Department of Pathology, Louisiana State University Medical Center, Shreveport. C. W. is currently supported by a fellowship from the Cancer Research Fund of the Damon Runyon-Walter Winchell Foundation.

The opinions or assertions contained herein are the private views of the authors and are not to be construed as official or as reflecting the views of the Department of the Army or the Department of Defense. This is a U.S. government work; there are no restrictions on its use.

Accepted for publication March 16, 2000.

Dr. Washington's current address: Hamon Cancer Center, University of Texas Southwestern, 5323 Harry Hines Blvd., Dallas, TX 75235-8593.

Address reprint requests to Dr. Jack H. Lichy, Molecular Pathology Division, Department of Cellular Pathology, Armed Forces Institute of Pathology, 14th St. and Alaska Ave. N.W., Washington, DC 20306-6000. E-mail: lichy@afip.osd.mil. 
of the malignancy or, instead, an indicator of an underlying global pathological process. For example, the identification of lobular carcinoma in situ in one breast is associated with an increased risk of cancer in either breast. Therefore, lobular carcinoma in situ seems to be an indicator for a predilection to develop cancer rather than a direct precursor of the tumor. ${ }^{10-12}$ The few studies that have analyzed genetic alterations in fibrocystic change have focused largely on atypical ductal hyperplasia; other morphological entities included under this heading have not been well characterized for genetic abnormalities. ${ }^{3,13-15}$

The purpose of the present study was to characterize the frequency of $\mathrm{LOH}$ in the components of fibrocystic change and to begin to address the question of whether benign proliferative lesions can be identified that show evidence for a genetic relationship with a carcinoma in the same breast. In this study, 32 cases of fibrocystic change were studied for $\mathrm{LOH}$ at 14 chromosomal loci. Individual foci of epithelial cells, representing normal TDLUs, ductal hyperplasia, adenosis, and apocrine metaplasia, were isolated from surrounding tissue by microdissection. LOH was observed at some frequency in each component analyzed, but was most often observed in this group of specimens in foci of apocrine metaplasia. Based on these findings, a group of breast carcinomas was studied for the presence of apocrine metaplasia foci adjacent to carcinoma. Genetic analysis of these foci provided evidence consistent with a developmental relationship between the apocrine metaplasia specimen and the adjacent tumor.

\section{Materials and Methods}

\section{Cases}

The surgical pathology archives at the Louisiana State University Medical Center-Shreveport were searched for cases with a diagnosis of fibrocystic disease or fibrocystic change. Cases that contained carcinoma were excluded. Slides were reviewed to identify cases with dissectable foci of hyperplasia, adenosis, and apocrine metaplasia. A total of 32 cases were selected. Of these 32 cases, 24 were postmenopausal, two were premenopausal, and the menopausal status of the other six cases could not be ascertained. These cases are referred to with the designation FC followed by a case number to distinguish them from the breast cancer cases that were used in the second portion of the study presented in this report. The components of fibrocystic change were identified on hematoxylin and eosin-stained sections and then on unstained 12- $\mu \mathrm{m}$ sections after deparaffinization with Ameri-clear (Fisher). The individual foci were microdissected from surrounding tissue with a small scalpel blade. Because lymph nodes were not available for most of these cases, normal control specimens were taken from regions of the available sections rich in stromal or lymphoid cells. Tissue was placed in $75 \mu$ l of lysis buffer $(120 \mu \mathrm{g} / \mathrm{ml}$ proteinase $\mathrm{K}, 50 \mathrm{mmol} / \mathrm{L} \mathrm{KCl}, 10 \mathrm{mmol} / \mathrm{L}$ Tris (pH 8.0), $0.1 \mathrm{mmol} / \mathrm{L}$ EDTA, and 0.5\% Tween 20) and incubated at $55^{\circ} \mathrm{C}$ for $12-16$ hours, followed by $95^{\circ} \mathrm{C}$ for 5 minutes to inactivate the protease. Insoluble debris was pelleted by centrifugation, and the supernatant was used as the source of DNA template for polymerase chain reaction (PCR).

Cases used for the identification of apocrine metaplasia adjacent to carcinoma were from the archives of the Armed Forces Institute of Pathology and have been described previously. ${ }^{16}$

\section{PCR Conditions}

The panel of markers used consisted of a group of 14 dinucleotide repeat polymorphisms known to have a high heterozygote frequency and to show a high frequency of $\mathrm{LOH}$ in breast cancer. The markers in the panel, and the associated tumor suppressor genes when known, included Cl3-CA373 at chromosome 3p14, ${ }^{17}$ D9S171 and D9S1748 (p16 cdk inhibitor) ${ }^{18,19}$ at chromosome 9p; D11S4046 and TH (tyrosine hydroxylase) at $11 \mathrm{p} 15^{16,20}$; D13S260 (BRCA2) and D13S263 (RB1) at 13q12-1421; D16S496, D16S512, and D16S421 at16q2222; D17S1788 and D17S1880 and P53 at chromosome 17p; and D17S795 at chromosome $17 q^{20}$

$P C R$ reactions were performed in the presence of one ${ }^{32} \mathrm{P}$-end-labeled primer. PCR assays were performed in $20-\mu$ reactions containing $10 \mathrm{mmol} / \mathrm{L}$ Tris- $\mathrm{HCl}(\mathrm{pH}$ 8.3), $50 \mathrm{mmol} / \mathrm{L} \mathrm{KCl}, 1.5 \mathrm{mmol} / \mathrm{L} \mathrm{MgCl}_{2}, 1 \mathrm{U}$ of AmpliTaq Gold DNA Polymerase (Perkin-Elmer, Foster City, CA), 0.2 $\mathrm{mmol} / \mathrm{L}$ of each deoxynucleoside triphosphate, 0.2 $\mu \mathrm{mol} / \mathrm{L}$ primer, and $1 \mu \mathrm{l}$ of the tissue lysate. A 5 -minute $95^{\circ} \mathrm{C}$ denaturation step was followed by 40 cycles of $95^{\circ} \mathrm{C}$ for 30 seconds, $55-60^{\circ} \mathrm{C}$ for 30 seconds, $72^{\circ} \mathrm{C}$ for 30 seconds, and a 10 minute final extension step at $72^{\circ} \mathrm{C}$. Reactions were stopped by the addition of loading buffer (95\% formamide, $10 \mathrm{mmol} / \mathrm{L}$ EDTA, 0.1\% bromophenol blue, and $0.1 \%$ xylene cyanol). The PCR products were heat denatured for 5 minutes at $95^{\circ} \mathrm{C}$ and loaded on a $6 \%$ acrylamide/7 mol/L urea gel. Results were visualized by autoradiography for $2 \mathrm{~h}$ to overnight at room temperature with Kodak XAR-5 film. In addition, to permit quantitation of allele ratios, gels were exposed to storage phosphor screens, and images were acquired with a Molecular Dynamics Storm Imaging System. Bands were quantitated by using the Molecular Dynamics ImageQuant software package.

Results were considered informative if the normal control specimen revealed a heterozygous genotype. A change in allele ratio of more than $50 \%$ relative to the normal control was interpreted as $\mathrm{LOH}$.

\section{Results}

\section{$\mathrm{LOH}$ in Fibrocystic Change}

Thirty-two cases showing fibrocystic change were selected for this study. Components of fibrocystic change were isolated from these cases by microdissection, resulting in a total of 90 specimens. Microdissection permitted the isolation of highly purified populations of the 

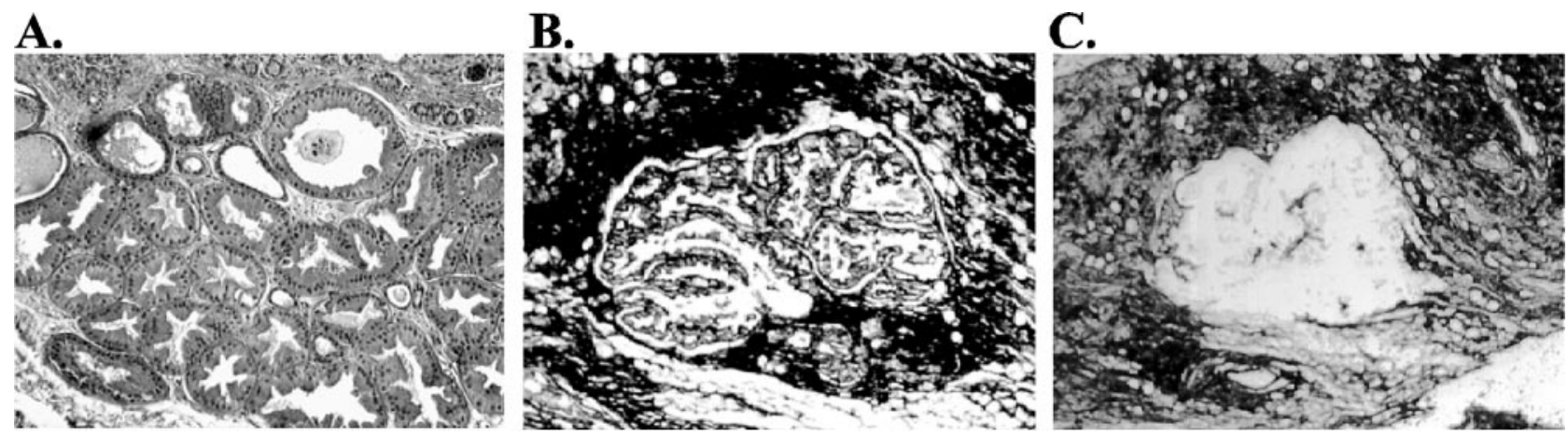

Figure 1. Histology and microdissection of apocrine metaplasia. A: Focus of apocrine metaplasia on hematoxylin and eosin-stained section. B: Appearance of apocrine metaplasia on an unstained tissue section before dissection. C: Appearance of same area after dissection.

cells of interest, as illustrated in Figure 1. The specimens included 27 morphologically normal TDLUs, 23 foci of adenosis, 19 of apocrine metaplasia, and 21 of ductal hyperplasia. Each of these specimens was analyzed for $\mathrm{LOH}$ at 14 dinucleotide repeat polymorphisms representing seven chromosomal arms. These markers were chosen to represent loci that show $\mathrm{LOH}$ with high frequency in breast cancer. ${ }^{23}$

Examples of $\mathrm{LOH}$ in these specimens are shown in Figure 2. The ductal hyperplasia specimen from case FC1 demonstrated $\mathrm{LOH}$ at four loci, including $13 q$ and $16 \mathrm{q}$ as shown in the figure. The apocrine metaplasia specimen from this case did not show $\mathrm{LOH}$. LOH in apocrine metaplasia is illustrated by cases $\mathrm{FC} 12$ at $9 p$ and $17 p$ markers, and by case FC46 at $13 q$ and $16 q$ markers. The results with the 17p marker D17S1880 for case FC46 show retention of heterozygosity. Examples of $\mathrm{LOH}$ detected in morphologically normal TDLUs are presented for cases FC56 and FC57, and an example of $\mathrm{LOH}$ in adenosis is shown for case FC55.

The $\mathrm{LOH}$ results summarized by type of lesion are presented in Table 1. The number of loci showing $\mathrm{LOH}$ are summarized in Table 2 by case number and specimen type. $\mathrm{LOH}$ was observed in 21 of the 32 cases with at least one microdissected specimen. In 18 of these 21 cases, $\mathrm{LOH}$ was observed in only one specimen of the several that were analyzed. The mean and range of patient ages did not differ significantly in cases with (mean 53.7, range 35-74) and without (mean 54.7, range 38 75) $\mathrm{LOH}$. Of the 90 microdissected specimens analyzed, 24 showed $\mathrm{LOH}$. However, 19 of these had LOH at more than one marker. Thus the $61 \mathrm{LOH}$ events were nonrandomly distributed, with more specimens having $\mathrm{LOH}$ at more than one marker than would be expected by chance $\left(P<0.001, \chi^{2}\right.$ test for fit to a Poisson distribution). Of the different morphological entities studied, $\mathrm{LOH}$ was observed in $22 \%$ of normal TDLUs (6/27), $17 \%$ of adenosis (4/23), $19 \%$ of hyperplasias (4/21), and $53 \%$ of apocrine metaplasia (10/19). The difference in LOH frequency between apocrine metaplasia and the other specimens was statistically significant $(P=0.002$, Fisher exact test). In case 50, the microdissected hyperplasia and apocrine metaplasia specimens shared $\mathrm{LOH}$ at five loci. This number of shared genetic lesions provides strong support for a common precursor cell in a lineage that developed into the two morphologically distinct epithelial foci.

Because $\mathrm{LOH}$ was found with high frequency in apocrine metaplasia in nonneoplastic cases, we hypothesized that a focus of apocrine metaplasia could sometimes derive from a genetically altered cell that also gave rise to an adjacent carcinoma. To test this hypothesis, a panel of 115 cases of carcinoma of the breast that had previously been studied for $\mathrm{LOH}^{16}$ were reviewed to identify tumors with adjacent apocrine metaplasia. Fourteen such cases were identified. The foci of apocrine metaplasia were isolated by microdissection and analyzed for LOH with the same panel of markers used on the fibrocystic change specimens. $\mathrm{LOH}$ was observed with at least one marker in seven of the 14 apocrine metaplasia specimens. Examples of $\mathrm{LOH}$ results with apocrine metaplasia specimens are presented in Figure 3. In case 113, the apocrine metaplasia specimen shared $\mathrm{LOH}$ with the adjacent tumor at markers on $16 q$ and $17 p$, but in addition showed LOH at $11 \mathrm{p}$, which was not detected in the tumor. Examples of shared $\mathrm{LOH}$ are shown for cases 50 and 52, and in case $93 \mathrm{LOH}$ at a $17 \mathrm{p}$ marker was detected in the apocrine metaplasia specimen but not in the tumor.

The data from the apocrine metaplasia specimens was compared to the allelotypic data from the adjacent carcinoma (Table 3). Loci showing $\mathrm{LOH}$ in only one of the two specimens are shown in bold type. Loci showing $\mathrm{LOH}$ that were found in the tumor but could not be analyzed in the apocrine metaplasia because of insufficient material are shown in parentheses. The results from all seven cases are consistent with a genetic relationship between the apocrine metaplasia and its associated carcinoma, in that the specimens share $\mathrm{LOH}$ at one or more loci. The evidence for such a genetic relationship is strongest for cases 113 and 93, which demonstrated common $\mathrm{LOH}$ at three loci. In two cases (52 and 97), the tumor and apocrine metaplasia specimen showed identical $\mathrm{LOH}$ patterns. In two other cases (16 and 50), the tumor showed $\mathrm{LOH}$ at the loci found to have $\mathrm{LOH}$ in the apocrine metaplasia but showed additional $\mathrm{LOH}$ at other loci. In three of the cases $(65,93$, and 113), several shared loci showing $\mathrm{LOH}$ were observed, but additional $\mathrm{LOH}$ 


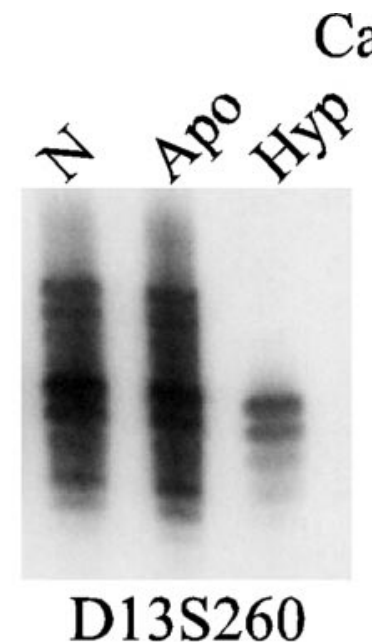

Case FC1

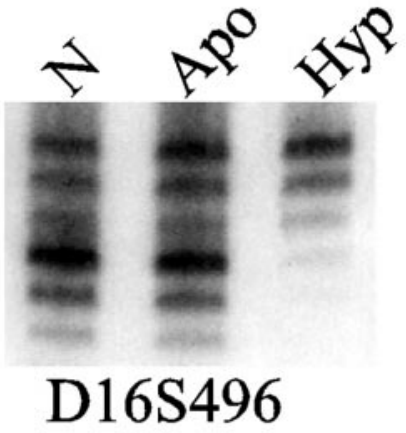

D16S496
Case FC12

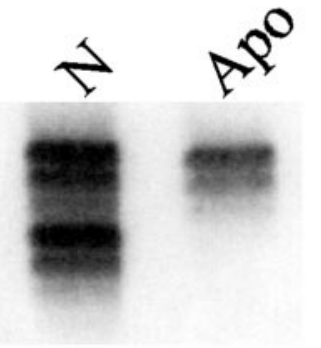

D9S171

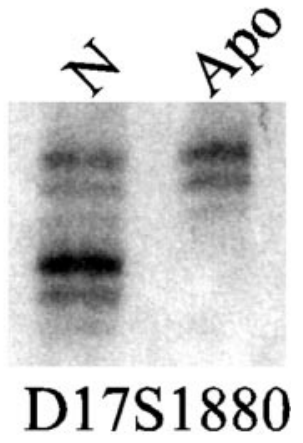

Case FC46

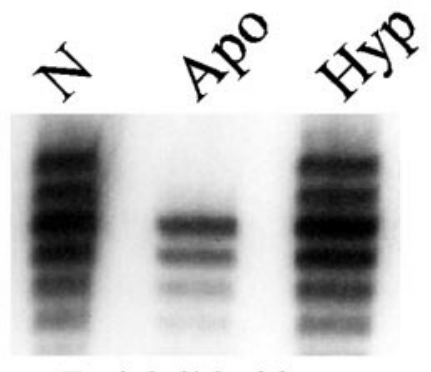

D13S260

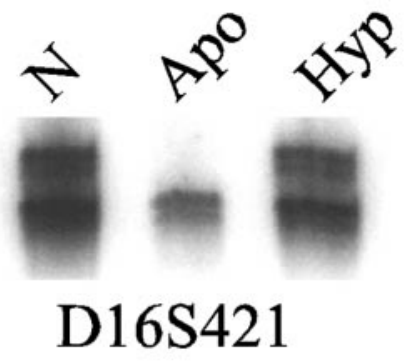

Case FC57

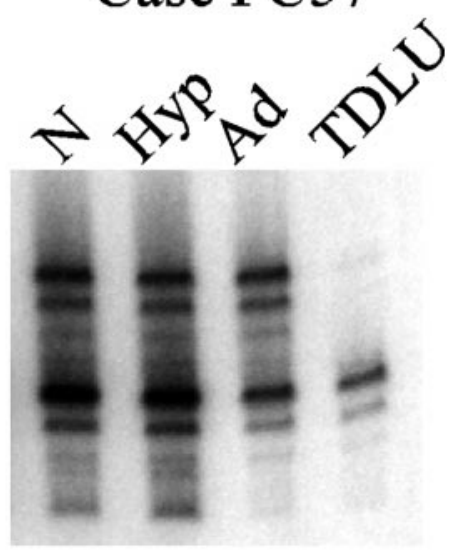

D17S1788

Figure 2. Examples of LOH in fibrocystic change. Results of PCR reactions for fibrocystic change cases at the indicated markers. N, normal control; Apo, apocrine metaplasia; Hyp, hyperplasia; Ad, adenosis; TDLU, morphologically normal terminal duct lobular unit.

was detected in the apocrine metaplasia specimen. The results are consistent with a pattern of growth involving the development of $\mathrm{LOH}$ at some loci in a cell that served as a common precursor for the apocrine metaplasia and the carcinoma.

\section{Discussion}

The morphological entities grouped together as fibrocystic change can be identified in more than $60 \%$ of premenopausal women and are often considered to be 
Table 1. LOH in Microdissected Fibrocystic Disease

\begin{tabular}{ll}
\hline \multicolumn{1}{c}{ Case no. } & \multicolumn{1}{c}{ Loci showing LOH } \\
\hline Normal specimens $(n=27)$ & \\
FC10 & $9 p, 13 q, 16 q, 17 p$ \\
FC23 & $16 q, 17 p$ \\
FC49 & $16 q, 17 p$ \\
FC52 & $9 p, 11 p, 16 q$ \\
FC56 & $11 p, 13 q, 16 q, 17 p$ \\
FC57 & $17 p$ \\
Adenosis $(n=23)$ & \\
FC16 & $13 q$ \\
FC24 & $17 p, 17 q$ \\
FC49 & $13 q$ \\
FC55 & $17 p, 17 q$ \\
Apocrine metaplasia $(n=19)$ & $9 p, 16 q$ \\
FC6 & $17 p$ \\
FC9 & $3 p, 9 p, 11 p, 16 q, 17 p$ \\
FC12 & $13 q, 16 q$ \\
FC13 & $3 p, 16 q$ \\
FC23 & $3 p, 9 p, 11 p$ \\
FC26 & $13 q, 16 q$ \\
FC46 & $11 p, 16 q$ \\
FC48 & $9 p, 16 q, 17 p, 11 p, 3 p$ \\
FC50 & $9 p, 11 p, 16 q$ \\
FC51 & $9 p, 13 q, 16 q, 17 p$ \\
Ductal hyperplasia $(n=21)$ & $3 p, 16 q$ \\
FC1 & $9 p$ \\
FC3 & $9 p, 11 p, 13 q, 16 q, 17 q$ \\
FC15 & \\
FC50 & \\
\hline
\end{tabular}

within the spectrum of normal physiological variation. ${ }^{8}$ Fibrocystic change is less common in postmenopausal women, a finding most likely resulting from the involution of the breast secondary to decreased hormonal stimulation. It has long been recognized that the presence of the proliferative components of fibrocystic change carries an increased relative risk for the subsequent development of

Table 2. Distribution of LOH Events*

\begin{tabular}{lcccc}
\hline Case & Normal & Hyperplasia & Adenosis & $\begin{array}{c}\text { Apocrine } \\
\text { metaplasia }\end{array}$ \\
\hline FC10 & 4 & $0,0^{\dagger}$ & - & 0 \\
FC49 & 2 & - & 1 & - \\
FC52 & 3 & 0 & - & - \\
FC56 & 4 & - & 0 & - \\
FC57 & 1 & 0 & 0,0 & - \\
FC1 & 0 & 4 & - & - \\
FC3 & 0 & 2 & - & 0 \\
FC15 & 0 & 1 & 0 & 5 \\
FC50 & 0 & 5 & 0 & - \\
FC16 & 0 & - & 1,0 & - \\
FC24 & 0 & - & 2 & 2 \\
FC55 & 0 & - & 2 & 1 \\
FC6 & 0 & 0 & 0 & 5 \\
FC9 & 0 & - & - & 2 \\
FC12 & 0 & - & - & 3 \\
FC13 & 0 & - & 0 & 2 \\
FC23 & 2 & 0 & - & 3 \\
FC26 & 0 & 0 & - & 0 \\
FC46 & 0 & 0 & - & \\
FC48 & 0 & - & 0 &
\end{tabular}

*Entries under each heading indicate the number of loci showing $\mathrm{LOH}$ for the specimen indicated.

†Two numbers are given when two distinct foci from the same case were analyzed. cancer. However, it is not known whether these proliferative lesions are direct precursors. Our data support the idea that the development of $\mathrm{LOH}$, a kind of genetic abnormality associated with cancer, can occur during the normal or hyperplastic growth of breast epithelial cells.

We have presented allelotype findings in a group of normal and benign proliferative lesions isolated from surrounding tissue elements by microdissection. Polymorphic markers were chosen to represent loci known to show $\mathrm{LOH}$ with high frequency in malignant tumors of the breast. ${ }^{24,25}$ These loci represent chromosomal arms 3p, $9 p, 11 p, 13 q, 16 q, 17 p$, and 17q. Our results revealed that even in normal breast epithelium and in the absence of cancer, $\mathrm{LOH}$ at one or more loci in our panel can be detected in approximately $20 \%$ of specimens. In these specimens, LOH was detected with significantly higher frequency in apocrine metaplasia than in hyperplasia, adenosis, or normal breast epithelium. To our knowledge, the allelotype of apocrine metaplasia has not previously been reported.

The occurrence of $\mathrm{LOH}$ in benign breast epithelium presented an opportunity to analyze a panel of previously characterized breast carcinomas for the genetic relationship between apocrine metaplasia and an adjacent carcinoma. Apocrine metaplasia is easily recognized by its distinct morphology, distinguishing it from adenosis and hyperplasia, the cells of which may show morphological patterns and cellular changes that make differentiation from carcinoma difficult. In theory there were four possible kinds of relationship between the apocrine metaplasia and the carcinoma. First, they could be completely unrelated, deriving from lineages that diverged before the development of any of the abnormalities that lead to the malignant phenotype. Second, the carcinoma could be a direct descendent of the apocrine metaplasia. In this case, the apocrine metaplasia would be expected to show some but not all of the genetic changes detected in the carcinoma. Third, the two specimens might derive from developmental pathways that diverged after some but not all of the observed genetic alterations occurred. Finally, it is possible that the apocrine metaplasia developed from the carcinoma. $\mathrm{LOH}$ analysis does not permit definitive resolution of this issue, primarily because it is not possible to prove that $\mathrm{LOH}$ of the same allele in two different specimens results from one event rather than two independent events that coincidentally give the same result. Nevertheless, the finding of two cases that show identical $\mathrm{LOH}$ at three loci provides supportive evidence for the concept that a common precursor cell containing genetic abnormalities can develop into both apocrine metaplasia and carcinoma. The possibility that this might occasionally occur via direct progression from apocrine metaplasia to carcinoma is supported by the $\mathrm{LOH}$ results in cases 16 and 50 .

The distribution of $\mathrm{LOH}$ events among the 90 specimens studied can be interpreted as evidence for a nonrandom occurrence of $\mathrm{LOH}$. The data set generated from the fibrocystic change cases implies at least 61 independent $\mathrm{LOH}$ events, counting $\mathrm{LOH}$ at more than one marker on the same chromosomal arm as one event. These 61 


\section{Case 113}

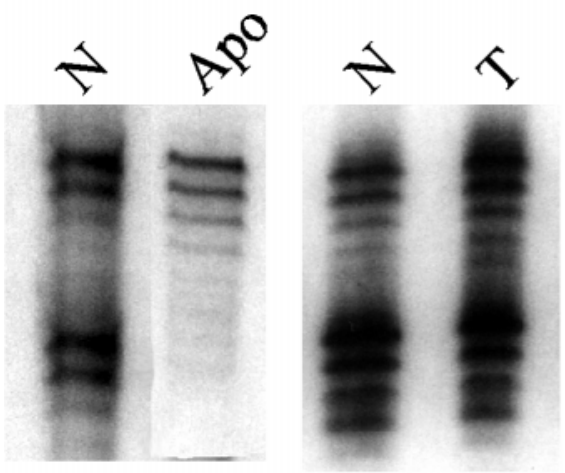

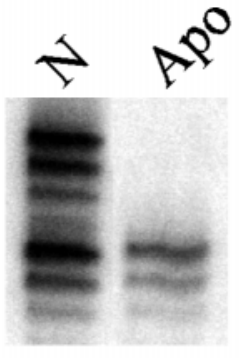

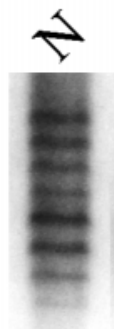

D16S496
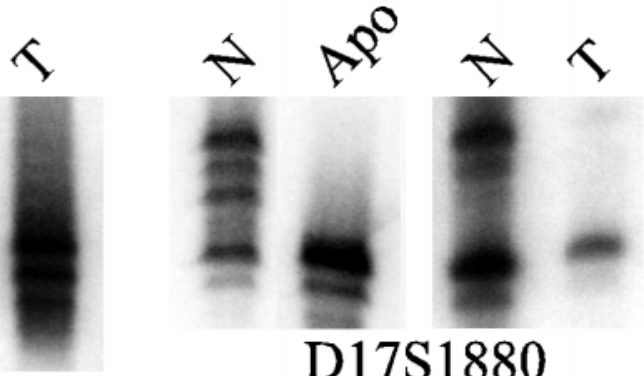

D17S 1880

\section{D11S4046}
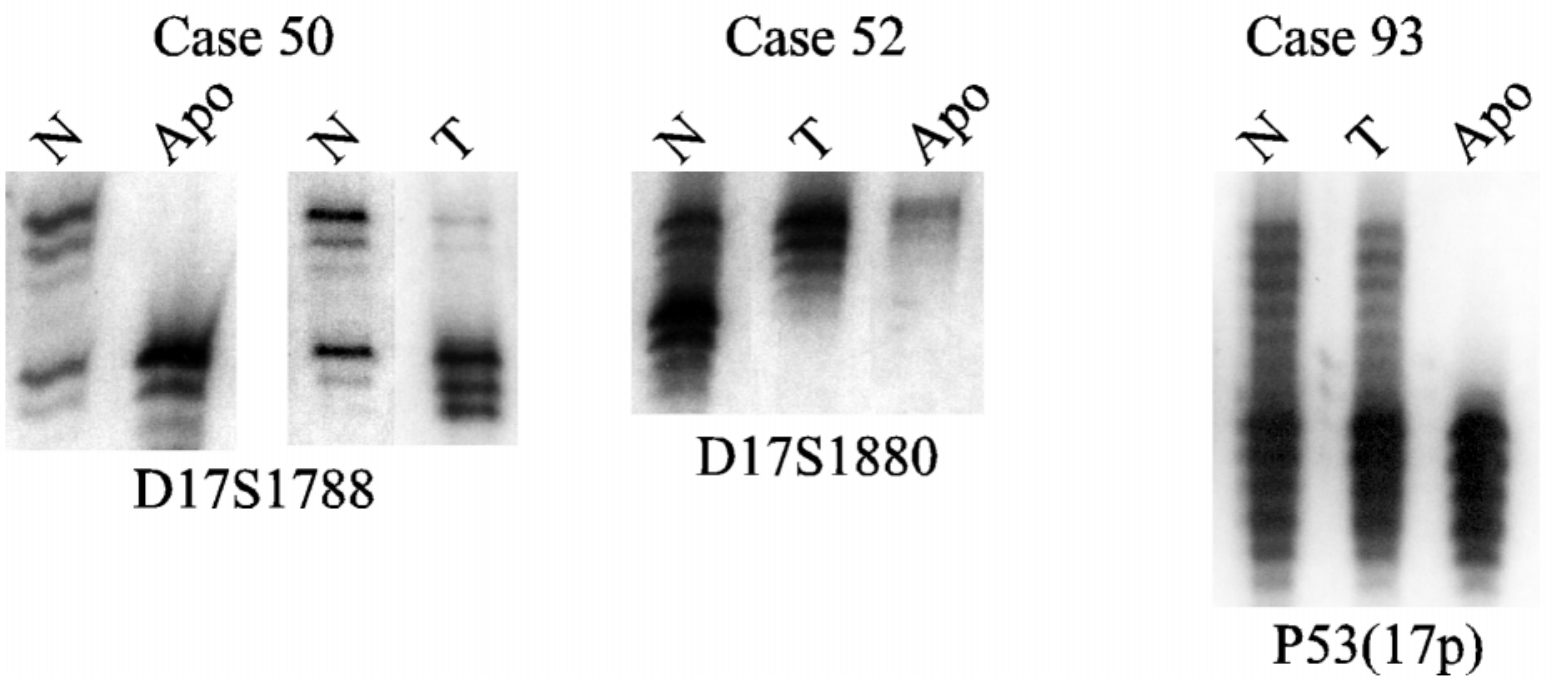

Figure 3. Comparison of LOH in apocrine metaplasia and adjacent tumor. Results of normal (N), apocrine metaplasia (Apo), and tumor (T) specimens from breast cancer cases are shown.

events are not distributed equally among the 90 specimens, but are clustered together so that only 24 specimens demonstrated LOH at any of the markers. However, of the specimens that did show $\mathrm{LOH}$, only five had $\mathrm{LOH}$ detected at only one marker. Thus there was a large excess of specimens with no $\mathrm{LOH}$ and with more than

Table 3. $\mathrm{LOH}$ in Apocrine Metaplasia and Adjacent Carcinoma

\begin{tabular}{rll}
\hline Case & \multicolumn{1}{c}{ Ap. met. } & \multicolumn{1}{c}{ Tumor } \\
\hline 16 & $11 p, 16 q$ & $9 p, 11 p, 16 q, 17 p, 17 q^{*}$ \\
50 & $17 p$ & $(11 p),{ }^{+}(13 q), 16 q, 17 p$ \\
52 & $17 p, 17 q$ & $(13 q), 17 p, 17 q$ \\
65 & $3 p, 9 p, 13 q, 17 q$ & $9 p, 13 q$ \\
93 & $3 p, 16 q, 17 p, 17 q$ & $3 p,(13 q), 16 q, 17 q$ \\
97 & $17 p$ & $17 p$ \\
113 & $11 p, 13 q, 16 q, 17 p$ & $13 q, 16 q, 17 p$ \\
\hline
\end{tabular}

*Loci showing $\mathrm{LOH}$ in apocrine metaplasia or tumor but not both are shown in bold.

${ }^{+}$Loci that show $\mathrm{LOH}$ in the tumor but could not be analyzed in the apocrine metaplasia because of insufficient material are in parentheses. one locus showing $\mathrm{LOH}$ relative to what would be expected if the distribution of $\mathrm{LOH}$ events were random. We therefore interpret our results as suggesting that epithelial foci that show LOH have acquired a more general deficiency in the maintenance of chromosomal integrity. It appears that in the breast such a deficiency can arise not only during the development of the components of fibrocystic change, but even during the normal formation of the terminal duct lobular units.

The results of this study confirm the finding that $\mathrm{LOH}$ can occur in morphologically normal TDLUs and in components of fibrocystic change. The results further demonstrate that $\mathrm{LOH}$ occurs commonly in apocrine metaplasia and that LOH patterns in some cases suggest a common clonal precursor to apocrine metaplasia and an adjacent carcinoma. Finally, we observed an apparent clustering of genetic alterations in a nonrandom manner, suggesting that foci of cells emerge both during normal development and in fibrocystic changes that have acquired deficiencies in chromosomal maintenance, resulting in a high frequency of $\mathrm{LOH}$. 


\section{References}

1. Deng G, Lu Y, Zlotnikov G, Thor AD, Smith HS: Loss of heterozygosity in normal tissue adjacent to breast carcinomas. Science 1996, 274: 2057-2059

2. O'Connell P, Pekkel V, Fuqua SA, Osborne CK, Clark GM, Allred DC: Analysis of loss of heterozygosity in 399 premalignant breast lesions at 15 genetic loci. J Natl Cancer Inst 1998, 90:697-703

3. Lakhani SR, Collins N, Stratton MR, Sloane JP: Atypical ductal hyperplasia of the breast: clonal proliferation with loss of heterozygosity on chromosomes 16q and 17p. J Clin Pathol 1995, 48:611-615

4. Kuukasjarvi T, Tanner M, Pennanen S, Karhu R, Kallioniemi OP, Isola $\mathrm{J}$ : Genetic changes in intraductal breast cancer detected by comparative genomic hybridization. Am J Pathol 1997, 150:1465-1471

5. Chuaqui RF, Zhuang Z, Emmert-Buck MR, Liotta LA, Merino MJ: Analysis of loss of heterozygosity on chromosome 11q13 in atypical ductal hyperplasia and in situ carcinoma of the breast. Am J Pathol 1997, 150:297-303

6. Euhus DM, Maitra A, Wistuba II, Alberts A, Albores-Saavedra J, Gazdar AF: Loss of heterozygosity at $3 \mathrm{p}$ in benign lesions preceding invasive breast cancer. J Surg Res 1999, 83:13-18

7. Larson PS, de las Morenas A, Cupples LA, Huang K, Rosenberg CL: Genetically abnormal clones in histologically normal breast tissue. Am J Pathol 1998, 152:1591-1598

8. Kamel OW, Kempson RL, Hendrickson MR: In situ proliferative epithelial lesions of the breast. Pathology (Phila) 1992, 1:65-102

9. Bodian CA, Perzin KH, Lattes R, Hoffmann P, Abernathy TG: Prognostic significance of benign proliferative breast disease. Cancer 1993, 71:3896-3907

10. Page DL, Kidd TE, Jr, Dupont WD, Simpson JF, Rogers LW: Lobular neoplasia of the breast: higher risk for subsequent invasive cancer predicted by more extensive disease. Hum Pathol 1991, 22:12321239

11. Rosen PP, Kosloff C, Lieberman PH, Adair F, Braun DW, Jr: Lobular carcinoma in situ of the breast: detailed analysis of 99 patients with average follow-up of 24 years. Am J Surg Pathol 1978, 2:225-251

12. Wheeler JE, Enterline HT, Roseman JM, Tomasulo JP, Mcllvaine CH, Fitts WT, Jr., Kirshenbaum J: Lobular carcinoma in situ of the breast: long-term follow-up. Cancer 1974, 34:554-563

13. Rosenberg CL, de las Morenas A, Huang K, Cupples LA, Faller DV, Larson PS: Detection of monoclonal microsatellite alterations in atypical breast hyperplasia. J Clin Invest 1996, 98:1095-1100
14. Rosenberg CL, Larson PS, Romo JD, De Las Morenas A, Faller DV: Microsatellite alterations indicating monoclonality in atypical hyperplasias associated with breast cancer. Hum Pathol 1997, 28:214-219

15. Kasami M, Vnencak-Jones CL, Manning S, Dupont WD, Page DL: Loss of heterozygosity and microsatellite instability in breast hyperplasia: no obligate correlation of these genetic alterations with subsequent malignancy. Am J Pathol 1997, 150:1925-1932

16. Lichy J, Zavar M, Tsai M, O'Leary T, Taubenberger J: Loss of heterozygosity at chromosome $11 \mathrm{p} 15$ during histological progression of ductal carcinoma of the breast. Am J Pathol 1998, 153:271-278

17. Jones MH, Yamakawa K, Nakamura Y: Isolation and characterization of 19 dinucleotide repeat polymorphisms on chromosome $3 p$. Hum Mol Genet 1992, 1:131-133

18. An HX, Niederacher D, Picard F, van Roeyen C, Bender HG, Beckmann MW: Frequent allele loss on 9p21-22 defines a smallest common region in the vicinity of the CDKN2 gene in sporadic breast cancer. Genes Chromosomes Cancer 1996, 17:14-20

19. Brenner AJ, Aldaz CM: Chromosome 9p allelic loss and p16/CDKN2 in breast cancer and evidence of p16 inactivation in immortal breast epithelial cells. Cancer Res 1995, 55:2892-2895

20. Dib C, Faure S, Fizames C, Samson D, Drouot N, Vignal A, Millasseau P, Marc S, Hazan J, Seboun E, Lathrop M, Gyapay G, Morissette J, Weissenbach J: A comprehensive genetic map of the human genome based on 5,264 microsatellites. Nature 1996, 380:152-154

21. Hamann U, Herbold C, Costa S, Solomayer EF, Kaufmann M, Bastert G, Ulmer HU, Frenzel H, Komitowski D: Allelic imbalance on chromosome 13q: evidence for the involvement of BRCA2 and RB1 in sporadic breast cancer. Cancer Res 1996, 56: 1988-1990

22. Filippova GN, Lindblom A, Meincke LJ, Klenova EM, Neiman PE, Collins SJ, Doggett NA, Lobanenkov VV: A widely expressed transcription factor with multiple DNA sequence specificity, CTCF, is localized at chromosome segment 16q22.1 within one of the smallest regions of overlap for common deletions in breast and prostate cancers. Genes Chromosomes Cancer 1998, 22:26-36

23. Brenner AJ, Aldaz CM: The genetics of sporadic breast cancer. Prog Clin Biol Res 1997, 396:63-82

24. Callahan R, Cropp CS, Merlo GR, Liscia DS, Cappa AP, Lidereau R: Somatic mutations and human breast cancer: a status report. Cancer 1992, 69(Suppl 6):1582-1588.

25. Chen LC, Kurisu W, Ljung BM, Goldman ES, Moore DD, Smith HS Heterogeneity for allelic loss in human breast cancer. J Natl Cancer Inst 1992, 84:506-510 